OUTP-96-32P

17th June 1996

hep-th/9606102

\title{
D-BRANE RECOIL AND LOGARITHMIC OPERATORS
}

\author{
Ian I. Kogan, Nick E. Mavromatos' and John F. Wheater \\ Department of Physics, University of Oxford, \\ Theoretical Physics, \\ 1 Keble Road, \\ Oxford, OX1 3NP, UK
}

\begin{abstract}
We construct the pair of logarithmic operators associated with the recoil of a $D$-brane. This construction establishes a connection between a translation in time and a world-sheet rescaling. The problem of measuring the centre of mass coordinate of the $D$-brane is considered and the relation between the string uncertainty principle and the logarithmic operators is discussed.
\end{abstract}

\footnotetext{
${ }^{1}$ PPARC Advanced Fellow
} 
Recently it was suggested [1] that the world-sheet description of the collective coordinates of a soliton in string theory is given by logarithmic operators [2]. This suggestion was based on earlier observation made in [3] that logarithmic operators may correspond to hidden continuous symmetries, which in the string soliton case turn out to be the target space symmetries related to the zero modes (collective coordinates).

Soliton backgrounds in string theory have received recently much attention as a result of Polchinski's discovery [4] that in the Ramond-Ramond sector superstring solitons can be simply described by open strings on a disk with Dirichlet boundary conditions for the collective coordinates of the soliton. Such constructions are known as $D$ (irichlet)-branes, and they are believed to be related to ordinary closed string backgrounds by duality transformations [4].

An important aspect of a $D$-brane quantization is the incorporation of proper recoil effects during the scattering of closed string states off the $D$-brane background [5, 6, 7]. It has been argued in these papers that operators describing the recoil appear as a result of extra logarithmic divergences arising in the open string one-loop amplitudes describing target-space quantum corrections to the scattering of elementary string states off the $D$ -

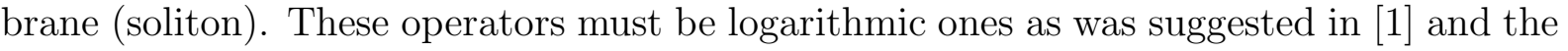
aim of this letter is to give a precise construction of the "recoil" logarithmic operators for a $D$-brane (actually we shall consider the 0-brane most of the time). As a consequence we will obtain a new and very interesting result concerning the connection between worldsheet scale and evolution in time as well as a new way to look at the string uncertainty relation. This might be relevant to the consistent quantization of soliton backgrounds in string theory [8].

It is important to stress that the logarithmic operators come in pairs, $C$ and $D$, and the OPE of the stress-energy tensor $\mathrm{T}$ with logarithmic operators $C$ and $D$ is non-trivial and involves mixing [2]

$$
\begin{aligned}
& T(z) C(w) \sim \frac{\Delta}{(z-w)^{2}} C(w)+\ldots \\
& T(z) D(w) \sim \frac{\Delta}{(z-w)^{2}} D(w)+\frac{1}{(z-w)^{2}} C(w)+\ldots
\end{aligned}
$$

where $\Delta$ is the conformal dimension of the operators and appropriate normalizaton of the $D$ operator has been assumed. The two point functions are given by [2], [3]

$$
\begin{aligned}
& <C(z) C(0)>\sim 0 \\
& <C(z) D(0)>\sim \frac{c}{|z|^{2 \Delta}} \\
& <D(z) D(0)>\sim \frac{c}{|z|^{2 \Delta}} \log |z| \quad c=\text { const }
\end{aligned}
$$

In the closed string case these relations are to be understood as being accompanied by 
their anti-holomorphic counterparts, whilst in the open string case (relevant here) $z, w$ are real parameters defined on the boundary of the world-sheet disk.

The above relations are the consequence of the behaviour of the conformal blocks of the underlying conformal field theory, the latter being determined by the four-point functions of the theory. Conformal blocks in theories involving logarithmic operators exhibit logarithmic scaling violations on the world sheet due to logarithmic divergencies. The absence of double or higher logarithmic divergences implies the vanishing of the $C-C$ two-point function in (2). This is an important point to remember if one tries to guess the leading divergent behaviour of the (unknown) conformal blocks of the theory from knowledge of the logarithmic operators. The non-trivial mixing between $C$ and $D$ operators is, on the other hand, a characteristic non-trivial property of the Jordancell structure of theories involving logarithmic operators. In the string-soliton case it is this mixing that leads to logarithmic divergencies of the annulus amplitudes [1]. As we shall discuss later on this mixing is associated with the lack of unitarity of the effective low-energy theory, in which $D$-brane (quantum) excitations are ignored.

In this paper we shall concentrate for simplicity on the case of the 0-brane. The extension to $p$-branes is straightforward, except for a few subtleties which we will discuss at the end of the paper. We recall that the world-sheet boundary operator describing the excitation of a $D$-brane is (see [9] and references therein)

$$
\mathcal{V}_{D}=\int_{\partial \Sigma} y_{i} \partial_{n} X^{i}+u_{i} X^{0} \partial_{n} X^{i}
$$

where $n$ denotes the normal derivative on the boundary of the world sheet $\partial \Sigma$, which at tree level is assumed to have the topology of a disk of size $L ; X^{i}, i=1, \ldots 9$ denote the collective excitations of the brane satisfying Dirichlet boundary conditions on the world-sheet boundary while $X^{0}$ is the time and satisfies standard Neumann boundary conditions,

$$
X^{i}(\text { boundary })=0, i=1, \ldots, 9 \quad \partial_{n} X^{0} \text { (boundary) }=0
$$

The coefficient $u_{i}$ in (3) is the velocity of the 0 -brane (point particle), and $y_{i}$ is the initial position $\left(X^{0}=0\right)$ of the collective coordinates of the 0 -brane 2 . The operator (3) describes an 'eternally moving' (boosted) 0-brane. Alternatively (3) can be thought of as generating the action of the Poincaré group on the 0-brane with $y_{i}$ parametrizing translations and $u_{i}$ parametrizing boosts.

To describe recoil we need an operator which has non-zero matrix elements between two different states of the 0-brane. In an impulse approximation this can be achieved by introducing a factor of the Heavyside function, $\Theta\left(X^{0}\right)$ into (3). This describes a 0-brane

\footnotetext{
${ }^{2}$ for flat Euclidean world-volume $y_{i}=\delta_{i j} y^{j}$. In this work we shall not discuss the important (and more realistic) case of curved world volumes, where the simple Dirichlet boundary conditions are known to be conformally non-invariant [4, 10].
} 
that starts moving at time $X^{0}=0$. The initial position of the 0 -brane at $X^{0}=0$ is assumed to be given by the $y_{i}$. One can then write down the following expression for the 'impulse' operator [6]:

$$
\left.\mathcal{V}_{i m p} \equiv \int d^{2} z \partial_{\alpha}\left(\left[u_{i} X^{0}\right] \Theta\left(X^{0}\right) \partial_{\alpha} X^{i}\right)=\int d \tau u_{i}\left(X^{0} \Theta\left(X^{0}\right)\right) \partial_{n} X^{i}\right) ; \quad i=1, \ldots 9
$$

where the coupling constant $u_{i}$ is the change in velocity of the brane, not only by physical arguments, but also as a result of imposing overall conformal invariance of the annulus and disc amplitudes (for more details see [5] - [7]). We note that the $\partial_{n} X^{i}$ parts yield the standard $1 / z^{2}$ world-sheet short-distance behaviour in (2). The non-trivial behaviour is encoded in the $X^{0}$ part, and, therefore, in what follows we shall concentrate on the $X^{0}$-dependent parts of (5).

Let us hypothesise that $X^{0} \Theta\left(X^{0}\right)$ plays the role of the $D$ operator. As it stands $\Theta\left(X^{0}\right)$ is ill-defined because $X^{0}$ is an operator so we define the integral representation

$$
\Theta_{\epsilon}\left(X^{0}\right)=-i \int_{-\infty}^{\infty} \frac{d q}{q-i \epsilon} e^{i q X^{0}} \quad, \quad \epsilon \rightarrow 0^{+}
$$

and then

$$
D_{\epsilon}=-i \int_{-\infty}^{\infty} \frac{d q}{q-i \epsilon} X^{0} e^{i q X^{0}}=-\int_{-\infty}^{\infty} \frac{d q}{(q-i \epsilon)^{2}} e^{i q X^{0}}
$$

where we have integrated by parts. To find the corresponding $C$ operator (1) we study the OPE of $D_{\epsilon}$ with the stress-energy tensor. Using the fact that the conformal dimension of the operator $e^{i q X^{0}}$ is $q^{2} / 2$ one easily gets

$$
\begin{aligned}
T(w) D_{\epsilon}(z) & =-\int_{-\infty}^{\infty} \frac{d q}{(q-i \epsilon)^{2}} \frac{q^{2}}{2(w-z)^{2}} e^{i q X^{0}} \\
& =-\frac{1}{(w-z)^{2}} \int_{-\infty}^{\infty} \frac{d q}{(q-i \epsilon)^{2}}\left[(q-i \epsilon)^{2}+2 i \epsilon(q-i \epsilon)-\epsilon^{2}\right] e^{i q X^{0}}
\end{aligned}
$$

In the first term there is no pole and after integration we get zero (formally it is a deltafunction of $X^{0}$ ), and the other two terms give $1 / q$ and $1 / q^{2}$ poles respectively so that

$$
T(w) D_{\epsilon}(z)=-\frac{\epsilon^{2} / 2}{(w-z)^{2}} D_{\epsilon}+\frac{1}{(w-z)^{2}} \epsilon \Theta\left(X^{0}\right)
$$

Comparing (9) with (11) we identify the $C$ operator to be $C_{\epsilon}=\epsilon \Theta_{\epsilon}\left(X^{0}\right)$ (note the factor of $\epsilon$ to which we will return). The action of the stress tensor on the operator $C_{\epsilon}$ is:

$$
T(w) C_{\epsilon}(z)=-i \epsilon \int_{-\infty}^{\infty} \frac{d q}{(q-i \epsilon)} \frac{q^{2} / 2}{\left.(w-z)^{2}\right)} e^{i q X^{0}(z)}
$$




$$
\begin{aligned}
& =-i \epsilon \frac{1}{2(w-z)^{2}} \int_{-\infty}^{\infty} \frac{d q}{q-i \epsilon}\left[(q-i \epsilon)^{2}+2 i \epsilon(q-i \epsilon)-\epsilon^{2}\right] e^{i q X^{0}} \\
& =-\frac{\epsilon^{2} / 2}{(w-z)^{2}} C_{\epsilon}
\end{aligned}
$$

as expected. These results show that the degenerate operators $C_{\epsilon}$ and $D_{\epsilon}$ have conformal dimension $\Delta=-\frac{\epsilon^{2}}{2}$ which is negative and vanishes in the limit $\epsilon \rightarrow 0^{+}$(note that this implies that the total dimension of the impulse operator (5), including the $\partial_{n} X^{i}$ factor, is $\left.1-\frac{\epsilon^{2}}{2}\right)$. We see that for non-zero $\epsilon$ the impulse operator is relevant in a renormalizationgroup sense. This is related to the very nature of the logarithmic operators which lie on the border line between conformal field theories and general two-dimensional field theories [1]- [3], [11]. It is the existence of such relevant deformations in the recoil problem that lead to a change of state of the 0-brane background [1, 8].

It is clear from (9) that we cannot work just with the $D_{\epsilon}\left(X^{0}\right)$ operator because $C_{\epsilon}\left(X^{0}\right)$ will necessarily be induced by a scale transformation. Thus, the proper recoil operator is described by

$$
\mathcal{V}_{r e c}=\int d \tau\left[y_{i} C_{\epsilon}\left(X^{0}\right) \partial_{n} X^{i}+u_{i} D_{\epsilon}\left(X^{0}\right) \partial_{n} X^{i}\right]
$$

where the coupling constants $y_{i}$ and $u_{i}$ in principle depend on scale. As we shall show in this article this scale dependence may be interpreted as providing a 'target time' dependent shift in the initial collective coordinates of the 0-brane, provided one identifies the worldsheet scale with an evolution parameter in target space.

We will now derive explicit expressions for the one and two point functions of the operators $D_{\epsilon}\left(X^{0}\right)$ and $C_{\epsilon}\left(X^{0}\right)$ appearing in (11) and verify that these operators have the correct properties to describe recoil. For calculational convenience we will analytically continue the target time $X^{0}$ to a Euclidean-signature field, and only at the end return to the Minkowskian signature. The two-point function on the world-sheet disc is given by

$$
\mathcal{G}_{z} \equiv<X^{0}(z) X^{0}(0)>\sim-2 \eta \log |z / L|^{2}
$$

where $L$ is the size of the disc, and $z$ parametrizes the world-sheet coordinates 1 . The value of $\eta$ depends on the (flat) target space signature of $X^{0}$, being +1 for Euclidean and -1 for Minkowski signature. The coincidence limit $z \rightarrow 0$ of (12) is regularized by an ultraviolet cut-off $a$ so that :

$$
\mathcal{G}_{0} \equiv<X^{0}(z) X^{0}(z)>\sim 2 \eta \alpha \quad \alpha \equiv \log |L / a|^{2}
$$

\footnotetext{
${ }^{3}$ If we map the disc on the upper half-plane, then $z$ becomes a real variable $\tau$ in the expression (12). This will be understood in what follows.
} 
To be rigorous one should consider the covariant propagator on the disc, of size $L$, and then take the coincidence limit. However, the simplified expression (13) is sufficient to yield the correct leading behaviour in the OPE.

Using (13) the one point function of the operator $C_{\epsilon}$ is given by

$$
\begin{aligned}
<C_{\epsilon}> & =-i \epsilon \int_{-\infty}^{\infty} \frac{d q}{q-i \epsilon}<e^{i q X^{0}}> \\
& =\epsilon^{2} \int_{-\infty}^{\infty} \frac{d q}{q^{2}+\epsilon^{2}} e^{-\eta q^{2} \alpha}
\end{aligned}
$$

The result of the integration in (14) may be expressed [12] in terms of the Error function $\operatorname{erf}(x) \equiv \frac{2}{\sqrt{\pi}} \int_{0}^{x} e^{-t^{2}} d t$ as:

$$
I=\int_{0}^{\infty} d x \frac{e^{-\alpha x^{2}}}{x^{2}+\epsilon^{2}}=\frac{\pi}{2 \epsilon} e^{\alpha \epsilon^{2}}(1-\operatorname{erf}(\epsilon \sqrt{\alpha})) \quad, \quad \operatorname{Re} \alpha>0
$$

Then,

$$
<C_{\epsilon}>=\epsilon \pi e^{\eta \epsilon^{2} \alpha}(1-\operatorname{erf}(\epsilon \sqrt{\eta \alpha}))
$$

We note, at this stage, that if we take the limit $\epsilon \rightarrow 0$ so that

$$
\epsilon^{2} \alpha=\epsilon^{2} \log |L / a|^{2}=\text { finite constant }
$$

then $\left\langle C_{\epsilon}>(14)\right.$ is zero for $\epsilon \rightarrow 0$. Similarly, the one point function for $D_{\epsilon}$ (11) is given by

$$
\begin{aligned}
<D_{\epsilon}> & =\int_{-\infty}^{\infty} \frac{d q}{(q-i \epsilon)^{2}}<e^{i q X^{0}}> \\
& =-i \frac{\partial}{\partial \epsilon} \int_{-\infty}^{\infty} \frac{d q}{q-i \epsilon} e^{-\eta q^{2} \alpha} \\
& =\frac{2}{\epsilon}\left\{-\pi \alpha \epsilon^{2} e^{\epsilon^{2} \alpha}(1-\operatorname{erf}(\epsilon \sqrt{\alpha}))+\epsilon \sqrt{\pi} \sqrt{\alpha}\right\}
\end{aligned}
$$

On account of (17), then, the one-point function (18) of the $D_{\epsilon}$ operator diverges as $1 / \epsilon$.

The two point function for $C_{\epsilon}$ is given by

$$
\begin{array}{r}
<C_{\epsilon}(z) C_{\epsilon}(0)>=-\epsilon^{2} \int_{-\infty}^{\infty} \int_{-\infty}^{\infty} \frac{d q d q^{\prime}}{(q-i \epsilon)\left(q^{\prime}-i \epsilon\right)}<e^{i q X^{0}(z)} e^{i q^{\prime} X^{0}(0)}> \\
=-\epsilon^{2} \int_{-\infty}^{\infty} \int_{-\infty}^{\infty} \frac{d q d q^{\prime}}{(q-i \epsilon)\left(q^{\prime}-i \epsilon\right)} \exp \left(-\left(q+q^{\prime}\right)^{2} \eta \alpha\right) \times \exp \left(2 \eta q q^{\prime} \log |z / a|^{2}\right)
\end{array}
$$


where we have used

$$
<e^{i q X^{0}(z)} e^{i q^{\prime} X^{0}(0)}>=e^{-\frac{q^{2}}{2}<X^{0}(z) X\left({ }^{0} z\right)>-\frac{q^{\prime 2}}{2}<X^{0}(0) X^{0}(0)>-q q^{\prime}<X^{0}(z) X^{0}(0)>}
$$

and (12,13). We now observe that in the limit $\alpha \rightarrow \infty$, with the condition (17) being assumed to hold, the first exponential would give a 'smeared' $\delta\left(q+q^{\prime}\right)$ contribution to the integral multiplied by a normalization factor $\sqrt{\pi / \alpha}$. Then the dominant contributions to the integral in (19) as $\alpha \rightarrow \infty$ come from the regions in $q^{\prime}$ integration for which $q^{\prime}=-q$. With this in mind we get

$$
\begin{aligned}
<C_{\epsilon}(z) C_{\epsilon}(0)> & \sim-\epsilon^{2} \sqrt{\frac{\pi}{\alpha}} \int_{-\infty}^{\infty} \frac{d q}{\left(q^{2}+\epsilon^{2}\right)} e^{-2 \eta q^{2} \log |z / a|^{2}} \\
& =-\epsilon^{2} \pi \sqrt{\frac{\pi}{\epsilon^{2} \alpha}} e^{2 \eta \epsilon^{2} \log |z / a|^{2}}\left(1-\operatorname{erf}\left(\epsilon \sqrt{2 \eta \log |z / a|^{2}}\right)\right) \\
& \stackrel{\epsilon \rightarrow 0}{\sim} 0+O\left(\epsilon^{2}\right)
\end{aligned}
$$

In a similar manner one may compute the $\left\langle C_{\epsilon}(z) D_{\epsilon}(0)>\right.$ function

$$
\begin{aligned}
<C_{\epsilon}(z) D_{\epsilon}(0)> & \sim-\frac{\epsilon}{2} \sqrt{\frac{\pi}{\alpha}} \frac{\partial}{\partial \epsilon} \int_{-\infty}^{\infty} \frac{d q}{q^{2}+\epsilon^{2}} e^{-2 \eta q^{2} \log |z / a|^{2}} \\
& =\frac{\pi}{2} \sqrt{\frac{\pi}{\epsilon^{2} \alpha}}\left\{e^{2 \eta \epsilon^{2} \log |z / a|^{2}}\left(1-4 \eta \epsilon^{2} \log |z / a|^{2}\right)\left(1-\operatorname{erf}\left(\sqrt{2 \eta \epsilon^{2} \log |z / a|^{2}}\right)\right)\right. \\
& \left.+2 \sqrt{\frac{2 \eta \epsilon^{2} \log |z / a|^{2}}{\pi}}\right\} \\
& \stackrel{\epsilon \rightarrow 0}{\sim} \frac{\pi}{2} \sqrt{\frac{\pi}{\epsilon^{2} \alpha}}\left(1-2 \eta \epsilon^{2} \log |z / a|^{2}\right)
\end{aligned}
$$

Finally the two-point function for $D_{\epsilon}$ is given by

$$
\begin{aligned}
<D_{\epsilon}(z) D_{\epsilon}(0)> & =\frac{1}{\epsilon^{2}}<C_{\epsilon}(z) D_{\epsilon}(0)> \\
& \stackrel{\epsilon \rightarrow 0}{\sim} \frac{\pi}{2} \sqrt{\frac{\pi}{\epsilon^{2} \alpha}}\left(\frac{1}{\epsilon^{2}}-2 \eta \log |z / a|^{2}\right)
\end{aligned}
$$

Thus we see that in the limit

$$
\epsilon \rightarrow 0, \quad \epsilon^{2} \log |L / a|^{2} \sim O(1)
$$

we obtain the canonical two-point correlation functions (21) with one exception - the singular $1 / \epsilon^{2}$ term in $\langle D D\rangle$. Note that the singularity structure at small $\epsilon$ of the correlation functions is unaffected by considering instead the connected correlation functions, as may easily be checked numerically. 
Because the exact value of the numerical constant in (24) is a free parameter we may choose it at will (the difference between different choices can be reabsorbed in the redefinition of the $\log z$ term) and thus establish an unambiguous relation between $\epsilon$, the regularization parameter in a target-space, and $L / a$, which is a world-sheet scale. Comparing with (12) it is most natural to put $\frac{1}{\epsilon^{2}}=2 \eta \log |L / a|^{2}$ and then we get (up to a normalization factor):

$$
\begin{aligned}
& <C_{\epsilon}(z) C_{\epsilon}(0)>\sim 0 \\
& <C_{\epsilon}(z) D_{\epsilon}(0)>\sim 1 \\
& <D_{\epsilon}(z) D_{\epsilon}(0)>\sim-2 \eta \log |z / L|^{2}
\end{aligned}
$$

Now let us make a scale transformation

$$
L \rightarrow L^{\prime}=L e^{t}
$$

which is really a finite size scaling (the only one which has physical sense for the open string world-sheet). Because of the relation between $\epsilon$ and $L$ this transformation will change $\epsilon$

$$
\epsilon^{2} \rightarrow \epsilon^{\prime 2}=\frac{\epsilon^{2}}{1+4 \eta \epsilon^{2} t}
$$

(note that if $\epsilon$ is infinitessimally small then $\epsilon^{\prime}$ is also infinitessimally small for any finite $t$ ) and we can deduce from the scale dependence of the correlation functions (25) that $C_{\epsilon}$ and $D_{\epsilon}$ transform as:

$$
\begin{aligned}
& D_{\epsilon} \rightarrow D_{\epsilon^{\prime}}=D_{\epsilon}-t C_{\epsilon} \\
& C_{\epsilon} \rightarrow C_{\epsilon^{\prime}}=C_{\epsilon}
\end{aligned}
$$

From this transformation one can see that the coupling constants in front of $C_{\epsilon}$ and $D_{\epsilon}$ in the recoil operator (11), i.e. the velocities $u_{i}$ and spatial collective coordinates $y_{i}$ of the brane, must transform like:

$$
u_{i} \rightarrow u_{i} \quad, \quad y_{i} \rightarrow y_{i}+u_{i} t
$$

Thus, in the presence of recoil a world-sheet scale transformation leads to an evolution of the $D$-brane in target space.

It is amusing that the pair of logarithmic operators describing $D$-brane recoil, can be used to give a new way to look at the stringy uncertainty principle [13]

$$
\Delta X_{i} \sim \frac{\hbar}{\Delta P_{i}}+O\left(\alpha_{s}^{\prime}\right) \Delta P_{i}
$$

where $\alpha_{s}^{\prime}$ is the string (or 0-brane) scale (involving the coupling constant of the string). Indeed, from (17) it becomes clear that small $\epsilon$ could be viewed as reflecting an uncertainty 
in the energy of the $D$ brane. The rôle of the $D$ operator in (11), then, can be easily checked to be in agreement with the standard quantum mechanical part of the uncertainty (30), whilst the $C$ operator is associated with essentially stringy effects. To see this one have to take into account that for finite $\epsilon$ the regulated $\Theta_{\epsilon} \sim \Theta\left(X^{0}\right) e^{-\epsilon X^{0}}$ and so $\epsilon=1 / \Delta t$, where $\Delta t$ is the resolution in time. The $X_{i}$ coordinate of the 0-brane starts to grow due to the recoil as $u_{i} X^{0}$ and the maximal value it will reach will be of order $u_{i} / \epsilon$ in the cases when $\epsilon \rightarrow 0^{+}$. To connect with the momentum uncertainty we have to show that $\Delta P_{i} \sim \hbar \epsilon / u_{i}$. Recall that to measure momentum in quantum theory (see, for example [14]) one has to scatter the quantum particle (0-brane in our case) on a "detector" and to measure both momentum and energy of "detector" before and after scattering. The "detector" may be a closed string state with definite energy and momentum. If the measurement (recoil) takes a finite time $\Delta t=1 / \epsilon$ the total energy is uncertain and one has the following relations

$$
\begin{array}{r}
p+P=p^{\prime}+P^{\prime} \\
\left|e+E-e^{\prime}-E^{\prime}\right| \sim \hbar \epsilon
\end{array}
$$

where small letters refer to the "detector" and big ones the 0-brane. Primed and unprimed letters correspond to the quantities before and after collision. Assuming that we know exactly the momentum and energy of the detector before and after the collision we obtain the uncertainties

$$
\Delta P=\Delta P^{\prime}, \quad\left(\Delta E-\Delta E^{\prime}\right) \sim \hbar \epsilon
$$

But

$$
\Delta E=\frac{\partial E}{\partial P} \Delta P=v \Delta P
$$

where $v$ is a velocity and so

$$
\left(v^{\prime}-v\right) \Delta P \sim \hbar \epsilon .
$$

In the case of the recoil operator (11) the change in velocity is $u$ and finally we get

$$
\Delta P \sim \hbar \epsilon / u
$$

i.e. precisely the contribution of the $D_{\epsilon}$ operator. However if one increases $\epsilon$ (making the uncertainty in momentum and energy bigger) one can see that the $C$ term will give a minimal bound of order of $y \epsilon$ (because of the factor of $\epsilon$ which appears in the definition of $C)$. Using (35) we see that the second term in (30) must be $\frac{u y}{\hbar} \Delta P$ and note that $u y$ has the same dimension as $\alpha_{s}^{\prime}$. Thus we see that the recoil term yields the usual Heisenberg part of the uncertainty $1 / \epsilon$, whereas the $C$ term is a stringy counterpart giving an $\epsilon$ term. It is important that, because of the C-D mixing, one can not evade the influence of the $C$ term. The fact that there are two different operators in the recoil operator enables us to reproduce both terms in (30). 
Let us discuss briefly how our results for 0-brane can be generalized for a $p$-brane. In this case one has $p+1$ coordinates $X^{I}, I=0,1, \ldots, p$ with Neuman boundary conditions and $(9-p)$ coordinates $X^{i}, i=p+1, \ldots 9$ satisfying Dirichlet boundary conditions

$$
\left.X^{i} \text { (boundary) }=0, i=p+1, \ldots, 9 \quad \partial_{n} X^{I} \text { (boundary }\right)=0, I=0,1, \ldots, p
$$

The relevant Poincaré symmetries are given now by translations $P^{i}$ in transverse $9-p$ directions and Lorentz rotations $M^{I j}$ which include boosts in transverse directions $M^{0 j}$ as well as rotations $M^{a j}, a=1, \ldots p ; j=p+1, \ldots, 9$ in transverse-longitudinal planes. Let us also note that the commutators of these generators

$$
\left[P^{i}, P^{j}\right]=0, \quad\left[P^{i}, M^{I j}\right]=g^{i j} P^{I}, \quad\left[M^{I i}, M^{J j}\right]=g^{I J} M^{i j}+g^{i j} M^{I J}
$$

corresponds to translations $P^{I}$ and rotations $M^{I J}$ inside the $p$-brane world-volume as well as rotations $M^{i j}$ in transverse planes, i.e. all these commutators do not change the state of the $p$-brane itself. This means that the result of two consecutive non-parallel recoils or bendings, with parameters $\left(y_{1}, u_{1}\right)$ and $\left(y_{2}, u_{2}\right)$ respectively, does not depend upon their ordering and is determined by the new set of parameters $\left(y_{1}+y_{2}, u_{1}+u_{2}\right)$ Besides recoil one may consider now the bending of the $p$-brane in which case instead of $\Theta_{\epsilon}\left(X^{0}\right)$ we have to consider $\Theta_{\epsilon}\left(X^{I}\right)$. The most general form of the "bending-recoil" operator will be

$$
\mathcal{V}_{\text {ben-rec }}=\int d \tau\left[y_{i I} C_{\epsilon}\left(X^{I}\right) \partial_{n} X^{i}+u_{i I} D_{\epsilon}\left(X^{I}\right) \partial_{n} X^{i}\right]
$$

where for coupling constants $y_{i I}$ and $u_{i I}$ we will get the same scaling relations (29).

It is curious to note that there is some similarity between the mechanism relating world-sheet scales to target time that we have described here and what occurs in the Liouville sector of the non-critical string theory [15, 16]. Although the $D$-brane calculation is formally in a critical string model the emergence of operators which are relevant for $\epsilon>0$ may indicate a connection with non-critical theories. Presumably it would be necessary to formulate the $D$-brane problem on a curved world volume \& in order to establish such a connection.

As a final comment, we wish to discuss the mixing between $C$ and $D$ operators in connection with the unitarity of the effective theory containing only low-energy point-like degrees of freedom. We shall concentrate on the computation of a four-point scattering amplitude in a generic string theory with logarithmic operators in its spectrum. For simplicity we shall concentrate in bosonic strings but one should note that string soliton backgrounds are known to be stable (exact) solutions of conformal field theory only in space-time supersymmetric theories. However the bosonic computation outlined here is indicative of the sort of modifications to the Veneziano amplitude (of the bosonic part of the superstring) in a generic theory with logarithmic operators. In the compactification of such string theories down to a four-dimensional flat space-time the logarithmic operators, 
which arise from the underlying conformal field theory models that describe the solitonic string background [1], are confined to the compact dimensions of the string, and one may assume that from a world-sheet point of view they lead to non-trivial 'bulk' contributions to the amplitudes. The issue we would like to address first is whether such compactifications are consistent with unitarity of the spectrum of the string. To be precise we shall perform the computation of correlation functions of four lowest-lying states in an uncompactified four-dimensional space time in an open (super)string theory with six dimensions compactified (a similar phenomenon takes place in the closed string sector but in this case there are logarithmic operators in both left and right sectors). The correlation function factorizes into an ordinary space-time part and a conformal block over the compact space

$$
A_{4}=<V_{1} V_{2} V_{3} V_{4}>_{M_{4}}<O_{1} O_{2} O_{3} O_{4}>_{K_{6}}
$$

where $M_{4}$ and $K_{6}$ indicate four-dimensional Minkowski and compactified space respectively. The existence of logarithmic operators in the compact sector would generically imply the presence of $\log |z|$ terms on the world-sheet coming from the anomalous operator product expansion of (degenerate) primaries. Indeed, consider the O.P.E. between two such operators of the full ten-dimensional theory, which we factorize into a non-compact piece $: e^{i k X}$ : and a compact piece $O_{i}$,

$$
: e^{i k X(z)}:: e^{i k^{\prime} X(0)}: O_{i}(z) O_{j}(0) \sim|z|^{k \cdot k^{\prime}-2} \frac{\log |z|}{|z|^{2}} e^{i\left(k+k^{\prime}\right) X} O \sim \partial_{k \cdot k^{\prime}-2}\left(|z|^{k \cdot k^{\prime}-2}\right) e^{i\left(k+k^{\prime}\right) X} O
$$

where for open strings the world-sheet variables are understood as lying on the real axis. Integrating the right-hand side around zero it is obvious that there exists a (non-unitary) double pole, thereby indicating the breakdown of tree-level unitarity in this type of compactification (!) This is an essentially stringy effect, with no precedent in $\sigma$-model computations.

From our discussion in this paper, this loss of unitarity seems to be only apparent in certain string backgrounds involving $D$-brane excitations. Indeed, if the compactified soliton backgrounds are viewed as $D$-branes, then in the above computation one should include quantum $D$-brane excitations. The latter produce themselves a mixing between boundary logarithmic operators, which in turn might cancel the mixing effects due to the logarithmic operators in the bulk. At present this problem is not solved.

\section{References}

[1] I.I. Kogan and N.E. Mavromatos, Phys. Lett. B375 (1996), 111; hep-th/9512210.

[2] V. Gurarie, Nucl. Phys. B410 (1993), 535. 
[3] J.S. Caux, I.I. Kogan and A. Tsvelik, Nucl. Phys. B466 (1996), 444; hep-th/9511130.

[4] J. Polchinski, Phys. Rev. D50 (1994), 6041 ; Phys. Rev. Lett., 75 (1995) 184, hepth/9510017;

J. Polchinski, S. Chaudhuri and C. Johnson, NSF-ITP-96-003 preprint, hepth/9602052, and references therein.

[5] W. Fischler, S. Paban and M. Rozali, Phys. Lett. B352 (1995), 298.

[6] V. Periwal and O. Tafjord, preprint PUPT-1607, hep-th/9603156.

[7] W. Fischler, S. Paban and M. Rozali, hep-th/9604014, Phys. lett. B to appear;

D. Berenstein, R. Corrado, W. Fischler, S. Paban and M. Rozali, preprint UTTG06-96, hep-th/9605168.

[8] J. Ellis, N.E. Mavromatos and D.V. Nanopoulos, preprint ACT-04/96, CERNTH/96-81, CTP-TAMU-11/96, OUTP-96-15P; hep-th/9605046, to appear in Int. J. Mod. Phys. A.

[9] C. Callan and I. Klebanov, Nucl. Phys. B465 (1996), 473; hep-th/9511173.

[10] M. Li, preprint BROWN-HET-1027, hep-th/9512042.

[11] M.R. Gaberdiel and H. G. Kausch, preprint DAMTP 96-36, hep-th/9604026;

M.A.I. Flohr, preprint IASSNS-HEP-96/54, hep-th/9605151.

[12] Tables of Integrals, Series and Products, I. Gradshteyn and I. Ryzhik (Academic Press, New York 1980).

[13] G. Veneziano, Europhys. Lett. 2 (1986), 199;

D. Amati, M. Ciafaloni and G. Veneziano, Phys. Lett. B216 (1989), 41;

K. Konishi, G. Paffuti and P. Provero, Phys. Lett. B234 (1990), 276.

[14] L.D. Landau and E.M. Lifshitz, "Quantum Mechanics (non-relativistic theory)", 3rd. edn., pub. Pergammon Press, section 16.

[15] I. Kogan, preprint UBCTP-91-13 (1991); Proc. Particles and Fields 91, p. 837, Vancouver 18-21 April 1991 (eds. D. Axen, D. Bryman and M. Comyn, World Sci. 1992); see also Phys. Lett. B265 (1991), 269.

[16] J. Ellis, N.E. Mavromatos and D.V. Nanopoulos, Phys. Lett. B293 (1992), 37; Lectures presented at the Erice Summer School, 31st Course: From Supersymmetry to the Origin of Space-Time, Ettore Majorana Centre, Erice, July 4-12 1993, published in Proc. Subnuclear Series Vol. 31, p.1 (World Scientific, Singapore 1994);

Mod. Phys. Lett. A10 (1995), 425; ibid. 1685; hep-th/9305117. 